\title{
Platelet-derived growth factor (PDGF)-C inhibits neuroretinal apoptosis in a murine model of focal retinal degeneration
}

\author{
Yujuan Wang ${ }^{1,2}$, Mones S Abu-Asab ${ }^{3}$, Cheng-Rong Yu ${ }^{4}$, Zhongshu Tang ${ }^{2}$, Defen Shen ${ }^{1}$, Jingsheng Tuo ${ }^{1}$, Xuri Li $^{2}$ \\ and Chi-Chao Chan ${ }^{1,3}$
}

Platelet-derived growth factor (PDGF)-C is a member of the PDGF family and is critical for neuronal survival in the central nervous system. We studied the possible survival and antiapoptotic effects of PDGF-C on focal retinal lesions in $\mathrm{Ccl}^{-/-} / \mathrm{Cx} 3 \mathrm{Cr}^{-1-}$ on $\mathrm{C} 57 \mathrm{BL} / 6 \mathrm{~N}\left[\mathrm{Crb}^{\mathrm{rd} d 8}\right]$ (DKO $\mathrm{rd} 8$ ) background mice, a model for progressive and focal retinal degeneration. We found no difference in transcript and protein expression of PDGF-C in the retina between DKO $r d 8$ mice and wild type (WT, C57BL/6N). Recombinant PDGF-CC protein (500 ng/eye) was injected intravitreally into the right eye of DKO rd8 mice with phosphate-buffered saline as controls into the left eye. The retinal effects of PDGF-C were assessed by fundoscopy, ocular histopathology, A2E levels, apoptotic molecule analysis, and direct flat mount retinal vascular labeling. We found that the PDGF-CC-treated eyes showed slower progression or attenuation of the focal retinal lesions, lesser photoreceptor and retinal pigment epithelial degeneration resulting in better-preserved photoreceptor structure. Lower expression of apoptotic molecules was detected in the PDGF-CC-treated eyes than in controls. In addition, no retinal neovascularization was observed after PDGF-CC treatment. Our results demonstrate that PDGF-C potently ameliorates photoreceptor degeneration via the suppression of apoptotic pathways without inducing retinal angiogenesis. The protective effects of PDGF-C suggest a novel alternative approach for potential age-related retinal degeneration treatment. Laboratory Investigation (2014) 94, 674-682; doi:10.1038/labinvest.2014.60; published online 7 April 2014

KEYWORDS: age-related macular degeneration; apoptosis; neuroprotection; platelet-derived growth factor-C; retina

Platelet-derived growth factor (PDGF)-C is a PDGF family member. ${ }^{1,2}$ It is a polypeptide of 345 amino acids with potent pleiotropic effects and functions as a homodimer, PDGF-CC. ${ }^{3}$ PDGF-C possesses a CUB (complement C1r/ C1s, Uegf, Bmp1) domain preceding the PDGF homology core domain. It is necessary for the CUB domain to be proteolytically removed to allow the PDGF core domain to bind to the PDGF receptor (PDGFR). ${ }^{1,4,5}$ PDGF has two receptors: PDGFR- $\alpha$ and PDGFR- $\beta$. PDGF-CC preferentially binds to the PDGFR- $\alpha \alpha$ homodimer but can also bind to the PDGFR- $\alpha \beta$ heterodimer when PDGFR- $\alpha$ and PDGFR- $\beta$ are coexpressed in the same cell. ${ }^{5-7}$

Although PDGF-C is a novel molecule discovered a decade ago, it has been found to be essential in many biological processes. It has been reported that $P d g f c$ knockout mice on a 129 background cannot survive the perinatal period because of feeding and respiratory difficulties. ${ }^{8}$ PDGF-C is abundantly expressed in multiple cell types, including endothelial cells, ${ }^{7,9}$ vascular smooth muscle cells, ${ }^{7,9,10}$ pericytes, and vascular fibroblasts. ${ }^{711}$ In addition, PDGF-C is highly expressed in the central nervous system, including the retina, ${ }^{12,13}$ brain, ${ }^{1,12}$ and spinal cord. ${ }^{14}$ As a broad-acting neuroprotective factor, PDGF-C has been shown to have pro-survival effects on neurons via both direct and indirect pathways. ${ }^{5}$ In the retina, specifically, PDGF-C exhibits protective effects on retinal ganglion cells and neurons in the inner/outer nuclear layers (INL/ONL) and regulates the expression of apoptotic and survival genes. ${ }^{12}$ It has been shown that PDGF-C also induces human retinal pigment epithelium (RPE) proliferation and migration in vitro. ${ }^{15}$

\footnotetext{
${ }^{1}$ Immunopathology Section, Laboratory of Immunology, National Eye Institute, National Institutes of Health, Bethesda, MD, USA; ${ }^{2}$ State Key Laboratory of Ophthalmology, Zhongshan Ophthalmic Center, Sun Yat-Sen University, Guangzhou, China; ${ }^{3}$ Histopathology Core, National Eye Institute, National Institutes of Health, Bethesda, MD, USA and ${ }^{4}$ Molecular Immunology Section, Laboratory of Immunology, National Eye Institute, National Institutes of Health, Bethesda, MD, USA Correspondence: Dr C-C Chan, MD, Immunopathology Section, National Eye Institute, Bldg. 10, Rm. 10N103, NIH/NEl, 10 Center Drive, Bethesda, MD 20892-1857, USA. E-mail: chanc@nei.nih.gov
} 
Moreover, PDGF-C is a potent angiogenic factor during development and under pathological conditions. Related studies have demonstrated that PDGF-C plays a role in promoting proliferation, survival, and migration of vascular smooth muscle cells, pericytes, and vascular endothelial cells and their progenitors. ${ }^{11,16-18}$ PDGF-C inhibition could effectively suppress both choroidal and retinal neovascularization under pathological conditions. ${ }^{18}$

Age-related macular degeneration (AMD) is a multifactorial neurodegenerative disease characterized by progressive degeneration of the photoreceptor/RPE complex primarily in the retinal macula. It has become the major cause of irreversible central vision loss in the elderly worldwide. ${ }^{19-21}$ As the photoreceptors and RPE are closely associated, a change in any single component of this complex could ultimately result in photoreceptor death. ${ }^{22}$ Previous evidence reveals a link between photoreceptor degeneration and AMD. ${ }^{23-25}$ Many clinical and experimental studies have linked AMD neurodegeneration to a variety of factors, including aging, oxidative stress, parainflammation, genetic predisposition, and environmental elements. ${ }^{26-32}$ In certain conditions, the photoreceptor/RPE complex is exposed to numerous stimuli, such as oxidants, cytokines, and/or neurotoxins, which ultimately contribute to AMD pathogenesis. ${ }^{33}$ In addition, studies have shown that apoptosis of RPE and photoreceptors has a vital role in geographic atrophy AMD. ${ }^{34,35}$

Given the reported survival effects of PDGF-C on neurons, the aim of this study was to evaluate the neuroprotective and antiapoptotic effects of PDGF-C on the retinal lesions in a $C c l 2^{-/-} / \mathrm{Cx} 3 \mathrm{crl}^{-/-}$on $\mathrm{C} 57 \mathrm{BL} / 6 \mathrm{~N}\left[\mathrm{Crbl}^{r d 8}\right]$ (DKO $r d 8$ ) background mouse model of progressive and focal retinal degeneration, mimicking certain features of human AMD in addition to typical retinal dystrophy/dysplasia secondary to $r d 8$ background. ${ }^{36,37}$

\section{MATERIALS AND METHODS}

\section{Animals and Treatment}

We generated the DKO $r d 8$ mouse as a model of progressive, focal retinal degeneration, ${ }^{38,39}$ mimicking certain features resembling human AMD lesions. ${ }^{36}$ In addition to the Ccl2/Cx3crl double knockout, the C57BL/6N mouse line has the crumbs-like 1 (Crb1) mutation in homozygous form. ${ }^{40}$ The DKO $r d 8$ mouse has earlier onset and higher penetrance than $C c l 2$ and $C \times 3 c r 1$ single-knockout strains. ${ }^{36,41}$ The DKO $r d 8$ mice and age-matched wild-type (WT, C57BL/ $\left.6 \mathrm{~N}\left[\mathrm{Crb}^{r d 8}\right]\right)$ mice were bred in-house. The mouse number in our study was determined by power analysis, considering the following factors: $P$ value, power, effects, and alternative hypothesis (two-tailed). All animal experiments were performed under protocols approved by the National Eye Institute's Institutional Animal Care and Use Committee and were in compliance with the Association for Research in Vision and Ophthalmology Statement for the Use of Animals in Ophthalmic and Vision Research. The right eye of 6-week-old DKO $r d 8$ mice were intravitreally injected with the core domain of recombinant His-tagged PDGF-CC protein ( $500 \mathrm{ng} / \mathrm{eye}$ ) diluted in phosphate-buffered saline (PBS). Left eye was injected with the vehicle control PBS and served as control.

\section{Retina Isolation}

The mouse retina was isolated from eyes of WT and DKO $r d 8$ mice at 6-8 weeks of age. In brief, experimental animals were killed and their eyes were enucleated. The globes were dissected free of periocular connective tissue, the anterior segment was removed, and the retina was dissected free from the underlying RPE-choroidal eyecups. The isolated retina was collected to measure mRNA and protein expressions of PDGF-C by quantitative reverse transcription-polymerase chain reaction ( $\mathrm{qRT}-\mathrm{PCR}$ ) and western blot, respectively.

\section{RNA Isolation and qRT-PCR}

Total RNA was isolated from the primary retina using an RNeasy Mini Kit (Qiagen, Hilden, Germany). One microgram of RNA was reverse transcribed with Superscript II RNase H Reverse Transcriptase (Invitrogen, Grand Island, NY, USA) to $20 \mu \mathrm{l}$ cDNA. qRT-PCR was performed on the resulting cDNA using Brilliant SYBR Green QPCR Master Mix (Stratagene, La Jolla, CA, USA). The comparative cycle threshold value method, representing log transformation, was used to establish relative quantification of the fold changes in gene expression using the ABI 7500 System (Applied Biosystems, Foster City, CA, USA). Each 25- $\mu \mathrm{l}$ reaction volume contained $2 \times$ PCR master mix (SYBR Green/Rox; SA Biosciences, Frederick, MD, USA), $0.4 \mu \mathrm{M}$ of each primer, and $1 \mu \mathrm{l} \mathrm{cDNA}$. The cDNA was amplified with specific primers for 40 cycles. $\beta$-Actin was used as an internal control. Primers of $\beta$-actin, pdgfc, Fas ligand (Fasl), Fas, B-cell CLL/lymphoma-2 (Bcl2) and Bcl2-associated X protein (Bax) were purchased from SA Biosciences (Frederick, MD, USA).

\section{Western Blot}

Preparation of whole-cell lysates and western blot analysis were performed as described previously. ${ }^{42}$ Cell extracts from mouse retina $(30 \mu \mathrm{g} /$ lane $)$ were fractionated on $4-12 \%$ gradient SDS-PAGE (Invitrogen). Antibodies used included PDGF-C (R\&D Systems, Minneapolis, MN, USA) and $\beta$-actin (Santa Cruz Biotechnology, Santa Cruz, CA, USA). Preimmune serum was used in parallel as controls, and signals were detected with a HRP-conjugated secondary antibody (Zymed Laboratories) using the ECL-PLUS system (Amersham, Arlington Heights, IL, USA). Assays were repeated in four WT and five DKO mice. Image-J software is used to measure the band intensity in pixels.

\section{Fundus Photography and Clinical Grading}

Fundoscopy was performed before injection and every month following the PDGF-CC protein injection. A Karl Storz 
veterinary otoendoscope (Karl Storz, Tuttlingen, Germany) coupled with a Nikon D90 digital camera was used for taking mouse fundus photographs. Mice were given intraperitoneal injection of ketamine $(1.4 \mathrm{mg} / \mathrm{mouse})$ and xylazine $(0.12 \mathrm{mg} /$ mouse) for systemic anesthesia and topical $1 \%$ tropicamide ophthalmic solution (Alcon, Fort Worth, TX, USA) for pupil dilation. We assigned lesion grades by comparing the same quadrant of fundus area in all 65 mice over the 2-month course. ${ }^{43,44}$ Progression was defined as a $>10 \%$ increase in the number of the retinal lesions $(+1)$, a $>50 \%$ increase in the lesion size in at least $1 / 3$ of the lesions $(+2),>5$ fused lesions or the appearance of $>2$ chorioretinal scars $(+3)$, and diffuse chorioretinal scars $(+4)$. Regression was defined as a $>10 \%$ decrease in the number of retinal lesions $(-1)$, a $>50 \%$ decrease in lesion size in at least $1 / 3$ of the lesions $(-2)$, a $>50 \%$ disappearance of retinal lesions $(-3)$, and total disappearance of retinal lesions $(-4) .{ }^{43} \mathrm{~A}$ masked observer conducted the grading.

\section{Direct Labeling and Visualization of Retinal Vessels in the Flat-Mounted Retina}

Blood vessels in the retina were directly labeled by cardiac perfusion of an aqueous solution containing $1,1^{\prime}$-dioctadecyl-3,3,3',3'-tetramethylindocarbocyanineperchlorate (DiI, D-282, Invitrogen/Molecular Probes, Carlsbad, CA, USA), a lipophilic dye that labels endothelial cell membranes as previously described ${ }^{45}$ In brief, DKO $r d 8$ mice were killed by carbon dioxide inhalation and the thoracic cavity opened to expose the heart. A volume of $100 \mu \mathrm{l}$ of DiI stock solution $(6 \mathrm{mg} / \mathrm{ml}$ in $100 \%$ ethanol) was dissolved in $5 \mathrm{ml}$ of diluent comprising $\mathrm{PBS}$ and $5 \%$ glucose in a ratio of 1:4. The resulting solution was applied via cardiac perfusion at a rate of $1-2 \mathrm{ml} / \mathrm{min}$. This was immediately followed by the perfusion of $5-10 \mathrm{ml}$ of PBS and $5-10 \mathrm{ml}$ of $4 \%$ paraformaldehyde at a rate of $1-2 \mathrm{ml} / \mathrm{min}$. The eyes were collected, and the neuroretina was dissected free from the underlying RPE-choroidal eyecups. These flat-mount preparations were mounted on glass slides in the mounting medium (Fluoromount; Sigma, St. Louis, MO, USA) with the ganglion cell layer uppermost. The slides were covered and examined under an Olympus FV1000 Confocal Scanning Microscope. Retinal z-stack scan was used to examine all the retinal vascular layers. The total scanned thickness was $50 \mu \mathrm{m}$, and slices within the stack were separated by a distance of $5 \mu \mathrm{m}$. Image-J software is used to measure the fluorescence intensity in pixels per whole retina in each image.

\section{A2E Extraction and Quantification}

[2,6-dimethyl-8-(2,6,6-trimethyl-1-cyclohexen-1-yl)-1E,3E,5E, 7E-octatetra-enyl]-1-(2-hydroxyethyl)-4-[4-methyl-6(2,6, 6-trimethyl-1-cyclohexen-1-yl) 1E,3E,5E,7E-hexatrienyl]-pyridinium(A2E) is a lipofuscin generated from the visual cycle flux of all-trans-retinol and is relevant to aging and AMD pathogenesis. ${ }^{46}$ Whole eyes were removed in a dark room under dim red light and homogenized. A2E was extracted with chloroform/methanol as previously described. ${ }^{47}$ The extracts dissolved in methanol were separated by highperformance liquid chromatography (HPLC, Agilent 1100 LC, Wilmington, DE, USA) and detected by ultraviolet light at a wavelength of $435 \mathrm{~nm}$. A gradient of $40-95 \%$ acetonitrile/ $\mathrm{H}_{2} \mathrm{O}$ in $0.1 \%$ trifluoracetic acid was used to elute A2E on a reverse-phase C18 column (Agilent, eclipse XD8-C18, $5 \mu \mathrm{m}$, $4.6 \times 150 \mathrm{~mm}$ ) at a flow rate of $1.0 \mathrm{ml} / \mathrm{min}$. A2E was quantified using external A2E standards. ${ }^{48}$

\section{Histopathology}

Eyes were fixed for $30 \mathrm{~min}$ in $4 \%$ gluteraldehyde followed by $10 \%$ formalin for at least $24 \mathrm{~h}$. Fixed eyes were embedded in methacrylate and serially sectioned in the vertical pupillaryoptic nerve plane. Each eye was cut through the vertical pupillary-optic nerve axis (P-O sections) into 4-6 sections and stained with hematoxylin and eosin. All lesions on the whole retina of each $\mathrm{P}-\mathrm{O}$ section were analyzed and examined under a light microscope.

\section{Transmission Electron Microscopy}

The mouse eyes were fixed in $2.5 \%$ gluteraldehyde, dehydrated, and embedded with Spurr's epoxy resin. Ultrathin sections $(90 \mathrm{~nm})$ were prepared and double stained with uranyl acetate and lead citrate and viewed using a JEOL JEM 1010 transmission electron microscope.

\section{Frozen Sections, TUNEL Assay and Immunohistochemistry}

Eyes were collected following euthanasia of mice two months after the intravitreal injection, snap frozen and embedded in Tissue-Tek OCT Compound (Sakura Finetek USA, Torrance, CA, USA). Serial sections were cut along the vertical pupillary optic nerve plane and stored on slides at $-70^{\circ} \mathrm{C}$. Frozen mouse eye slides were fixed in $4 \%$ paraformaldehyde for $15 \mathrm{~min}$, washed in PBS. The fixed slides were labeled using a terminal deoxynucleotidyl transferase dUTP nick end labeling (TUNEL) assay kit according to the manufacturer's instructions (Roche, Nutley, NJ, USA). In addition, the fixed slides were blocked in ICC buffer with 5\% normal goat or rabbit serum for $30 \mathrm{~min}$ at $4{ }^{\circ} \mathrm{C}$. Samples were incubated overnight with primary antibodies to the following antigens: FasL, 1:50 (Abcam, Cambridge, MA, USA); Fas, 1:200 (Santa Cruz); Bax, 1:200 (Santa Cruz); and Bcl2, 1:200 (Santa Cruz). After washing with ICC buffer, 4',6-diamidino-2-phenylindole dihydrochloride (DAPI, 1:1000, Invitrogen) and secondary antibodies conjugated to either Alexa-488 or Alexa-555 (1:400, Invitrogen) were added and incubated at room temperature for $1 \mathrm{~h}$. All the slides with immunohistochemical staining were examined under an Olympus FV1000 Confocal Scanning Microscope. Image-J software was used to measure the fluorescence intensity in pixels per area in each image and expressed as fluorescence intensity ratio. 


\section{Statistical Analysis}

Statistical analyses were performed using GraphPad Prism 5 (GraphPad Software, San Diego, CA, USA). The data of qRT-PCR, A2E, TUNEL assay, and fluorescence intensity were compared using paired $t$-test. The data of clinical fundus scores were compared using Mann-Whitney $U$-test. A $P$-value $<0.05$ was considered statistically significant.

\section{RESULTS \\ PDGF-C Expression in the Neuroretina is Comparable between WT and DKO rd8 Mice}

We compared protein and mRNA transcript levels of PDGF-C in the freshly isolated neuroretina from naive WT and DKO $r d 8$ mice. Both $P d g f_{\mathcal{C}}$ transcripts (Figure 1a) and PDGF-C protein (Figures $1 \mathrm{~b}$ and $\mathrm{c}$ ) were expressed in the retina of both $\mathrm{WT}$ and DKO $r d 8$ mice. No significant differences were measured between the two mouse strains.

\section{PDGF-C-Attenuated Focal Retinal Lesions of DKO rd8 Mice}

To test whether PDGF-C would positively affect the retinal lesions in the DKO mice, we administered purified recombinant PDGF-CC protein into the eyes of 65 mice in three independent experiments. The experimental replicates yielded similar data. Fundoscopy revealed that PDGF-CCtreated eyes at 2 months had significant improvement over PBS controls, with fewer and smaller deep retinal lesions (arrows, Figures 2a and b). Histopathologically, the PBSinjected retina showed focal photoreceptor degeneration,

a

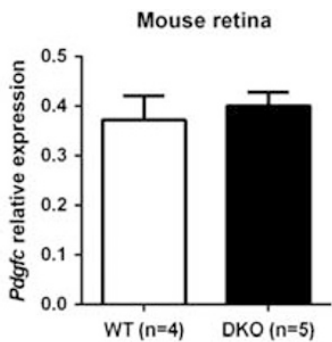

C

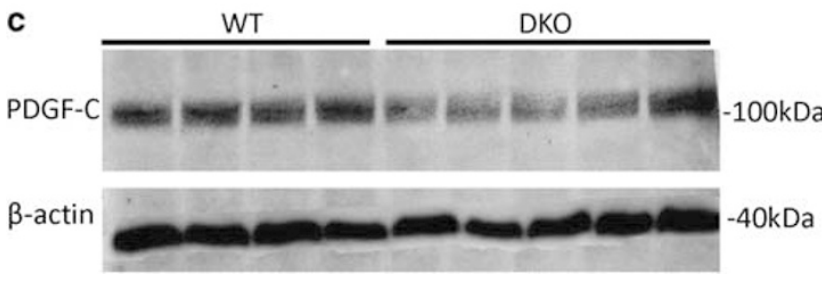

Figure 1 PDGF-DGF-C level in the retina of DKO rd8 and WT. (a) Pdgfc transcript levels in freshly isolated retina from WT $(n=4)$ than DKO rd8 $(n=5)$ mice using quantitative reverse transcription-polymerase chain reaction. Pdgfc transcript is comparably expressed between WT and DKO rd8 mice. (b) The PDGF-C protein ratio of western blot is calculated relative to $\beta$-actin (loading control). Image-J software is used to measure the band intensity in pixels. (c) Western blot detected PDGF-C expression in the retina in both WT and DKO rd8 mice. The size of molecular weight is given in $\mathrm{kDa}$. focal loss of inner/outer segment (IS/OS, Figure 2c, asterisks), and RPE vacuolation/pigmentary alteration in addition to the $r d 8$-associated photoreceptor dystrophic lesions in the outer plexiform layer (OPL, Figure 2c, arrows). In contrast, the PDGF-CC-treated retina presented fewer/smaller photoreceptor lesions, relatively healthy photoreceptor IS/OS, and normal RPE; however, most $r d 8$-associated focal photoreceptor dystrophic lesions remained (Figure 2c, arrows). A total of 25 pairs of eyes were histologically examined, 17/25 mice had AMD-like lesions and 23/25 mice had $r d 8$-associated lesions (Figure 2d). For AMD-like lesion comparison, 14 of $17(82.4 \%)$ mice showed fewer lesions in the PDGFCC-treated eyes compared with PBS-injected controls. For $r d 8$ lesion comparison, no statistical differences were found between the eyes injected with PDGF-CC and PBS. Moreover, PDGF-C treatment tended to reduce the levels of lipofuscin A2E, 11 of the 15 mice (below the dashed line) tested appeared to have decreased A2E, whereas the remaining four mice (above the dashed line) had increased expression (Figure 2e). To further investigate the protective effects of PDGF-C on mouse retinal vasculature, ultrastructural changes were compared. The pericytes of retinal vessels (asterisk) in PBS-injected eyes showed disintegration but were healthier in the PDGF-CC-injected retina (Figure 2f).

\section{PDGF-C has Antiapoptotic Effects on the Neuroretina of DKO rd8 Mice}

As a useful tool to detect DNA fragmentation of apoptotic cells, TUNEL assay showed less apoptotic cells in PDGF-CCtreated than PBS-injected retina in the ganglion cell layer, INL, and ONL (Figures $3 \mathrm{a}$ and $\mathrm{b}$ ). Several apoptosis-related molecules were measured to investigate whether PDGF-C could attenuate retinal lesions via apoptotic pathways. Between the PDGF-CC-treated and control eyes, Fasl (Figure 3c), Fas (Figure 3d), Bax (Figure 3e), and Bcl2 (Figure 3f) transcripts were compared between PDGF-CCtreated and PBS-injected eyes. The levels of Fas (Figure 3d) and Bax (Figure 3e) transcripts were significantly decreased in PDGF-CC-treated retina compared with the controls. Although there was an overall decrease in Fasl(Figure 3c) and $\mathrm{Bcl} 2$ (Figure 3f) in the PDGF-CC-treated retina compared with controls, the difference was not statistically significant. Immunohistochemistry showed less immunoreactivity against FasL in the ganglion cell layer, IPL, INL, and OPL (Figure $3 \mathrm{~g}$ ), Fas in the ganglion cell layer, IPL, INL, OPL, and IS/OS (Figure 3h), and Bax in the INL, OPL, and IS/OS (Figure $3 \mathrm{i}$ ), but higher expression of $\mathrm{Bcl} 2$ in the IPL, OPL, and IS/OS (Figure $3 \mathrm{j}$ ) in the PDGF-CC-treated retina than that in the PBS-injected retina.

\section{PDGF-C does not Induce Leakage and} Neovascularization in the Neuroretina of DKO rd8 Mice To further investigate whether PDGF-C would cause vascular leakage and vascularization in the retina of DKO $r d 8$ mice, retinal vasculature was examined using DiI staining. 

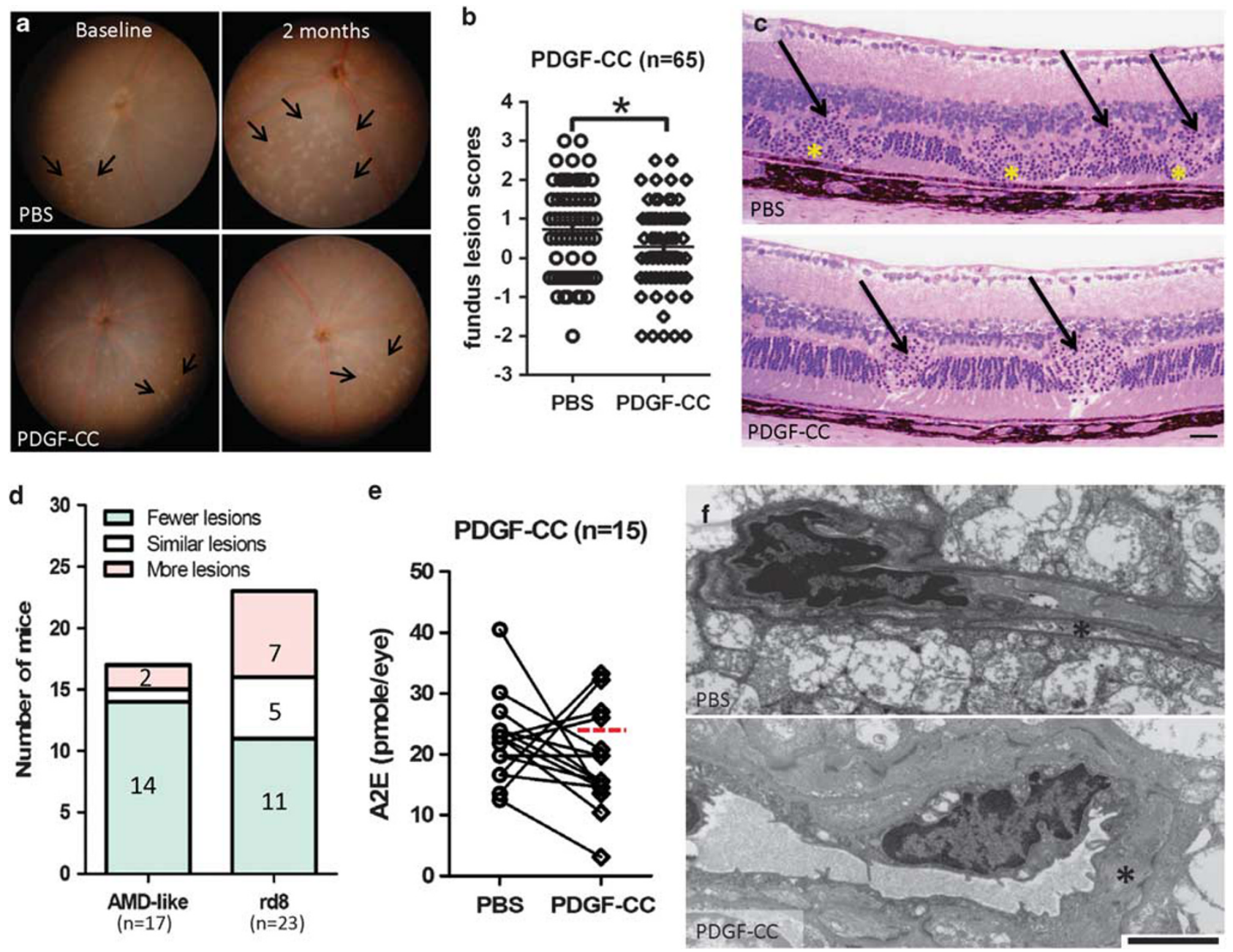

Figure 2 Retina-protective effect of PDGF-C on the DKO rd8 mouse retina. (a) The fundus of PBS-injected and PDGF-CC-treated DKO rd8 mouse eyes was taken by a Nikon D90 digital camera illuminated with a Karl Storz veterinary otoendoscope. Representative fundus findings showed changes in yellowish deep retinal lesions (arrows) in both eyes of the same mouse before and 2 months after PDGF-CC treatment. (b) Fundus scores of 65 pairs of eyes based on the fundus photographs showing altered progression scores between the two eyes at the end of 2 months after PDGF-CC treatment. (c) DKO rd8 mouse eyes were enucleated and embedded in methacrylate. Representative histopathological findings showed retinal lesions of both DKO (asterisks) and $r d 8$ (arrows)-associated lesions. Scale bar $=50 \mu \mathrm{m}$. (d) Histological comparison of AMD-like lesions and $r d 8$-associated lesions between each mouse.The lesions in the PDGF-CC-treated eye are compared with that in the PBS-injected eye of the same mouse. (e) PBS-injected and PDGF-CC-treated DKO $r d 8$ mouse eyes were enucleated and homogenized for A2E extraction with chloroform/methanol $(n=15)$. The 11 eyes below the dashed line showed decreased A2E. (f) DKO rd8 mouse eyes were enucleated and embedded with Spurr's epoxy resin. PDGF-C maintained pericytes (asterisks, scale bar $=2 \mu \mathrm{m}$ ) in retinal tissues when compared with PBS-injected eyes. ${ }^{*} P<0.05$.

Differences in vascular neovascularization were noted between PDGF-CC-treated and PBS-injected mice, the sizes and amount of vascular leakages were moderately smaller and less in the eyes that received PDGF-CC (Figure 4).

\section{DISCUSSION}

This study demonstrates that PDGF-C level is equally expressed in the retina of DKO $r d 8$ and WT mice. Local administration of recombinant PDGF-CC protein ameliorates focal photoreceptor degeneration in this mouse model with focal retinal degeneration. PDGF-CC-treated eyes showed fewer clinical and histopathological lesions in the retina. More importantly, the neuroprotective effect of
PDGF-C is associated with antiapoptotic activities on retinal neuronal cells. Molecular studies demonstrated decreased apoptosis with exogenous PDGF-CC compared with PBS controls. In addition, the used dosage of PDGF-CC that is effective for neuroprotection also can protect retinal vessel pericytes but can not induce retinal angiogenesis.

PDGF-C is widely expressed in multiple cell types and displays broad neuroprotective activities., ${ }^{7-11}$ Inside the eye, it has protective effects on retinal ganglion cells and INL/ONL neurons in mouse models with optic nerve crush and $\mathrm{N}$-methyl-D-aspartic acid (NMDA) injury. ${ }^{12}$ Given the neuroprotective and antiapoptotic effects of PDGF-C, we propose that intravitreal administration of the PDGF-CC 

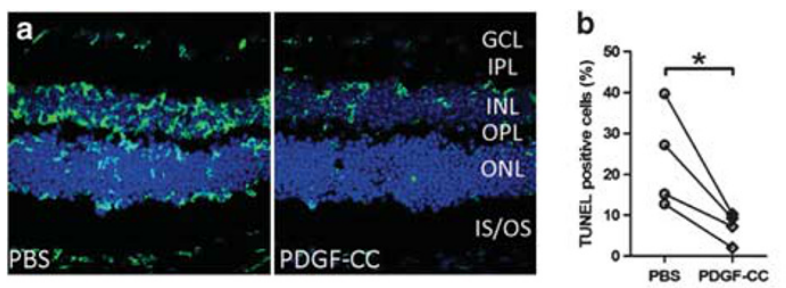

C

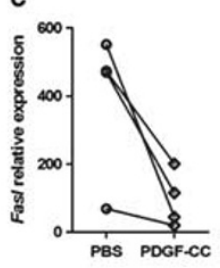

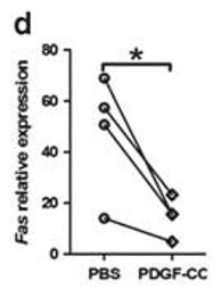
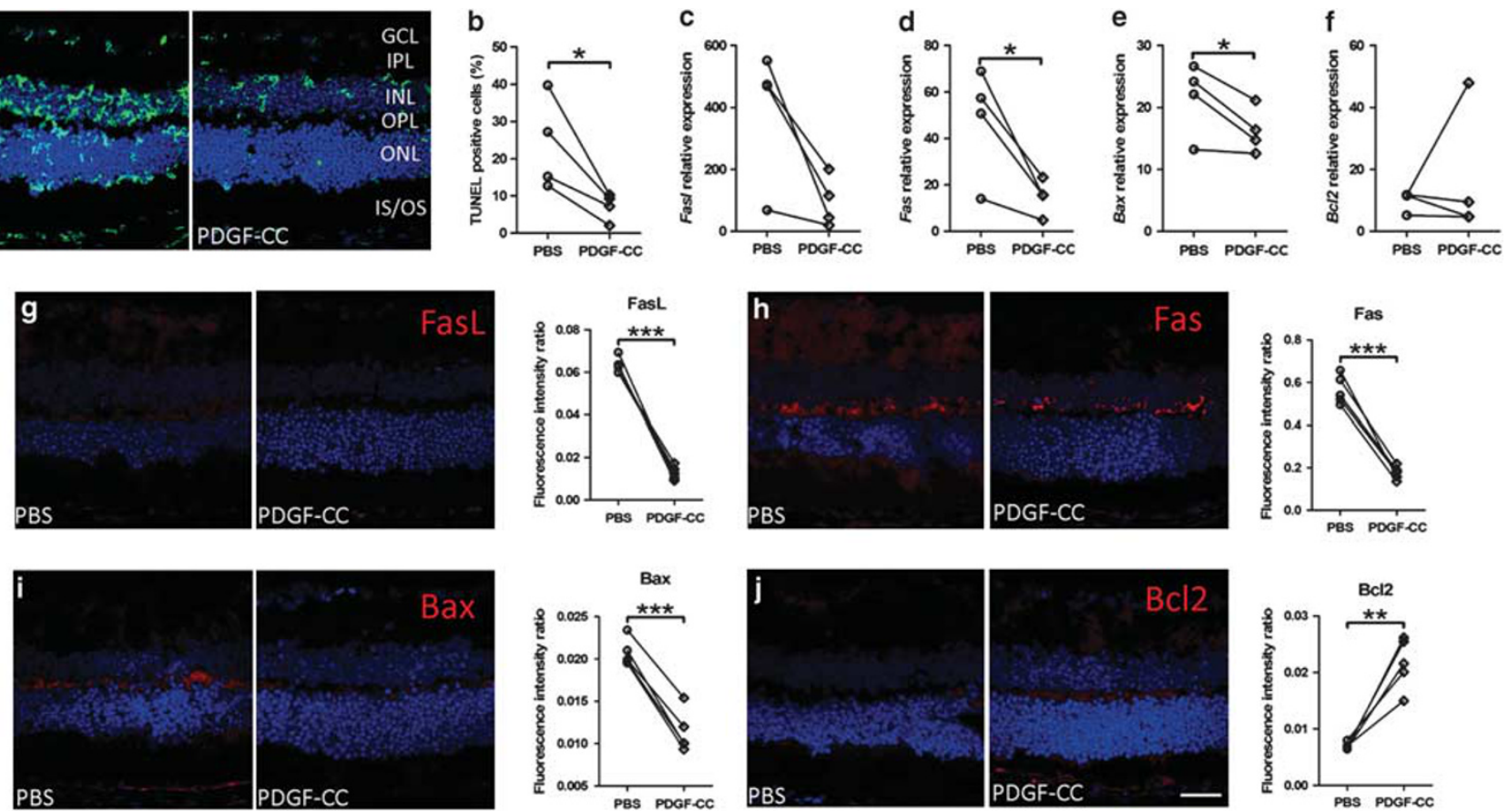

Figure 3 Antiapoptotic effects of PDGF-C on the neuroretina in DKO rd8 mice. (a) The frozen sections of PBS-injected and PDGF-CC-treated DKO rd8 eyes were stained with TUNEL assay (green color, $n=4$ ). (b) The percentage of TUNEL-positive cells per total cells in the neuroretina is calculated by Image-J software $(n=4)$. (c-f) The whole retina of PBS-injected and PDGF-CC-treated DKO $r d 8$ eyes was collected, and total RNA was isolated and converted to CDNA. Quantitative reverse transcription-polymerase chain reaction analysis was then performed to determine the levels of targeted gene transcripts as follows: (c) Fasl, (d) Fas, (e) Bax, and (f) $B C I 2(n=4)$. The frozen sections of the PBS-injected eye with thinner and irregular ONL and the PDGF-CC-treated DKO $r d 8$ eyes with relatively intact ONL were stained with apoptosis-related antibodies and immunoreactivity (red color) were evaluated under an Olympus FV1000 Confocal Scanning Microscope. Image-J software is used to measure the fluorescence intensity in pixels per area in each image and expressed as fluorescence intensity ratio. The same molecules were analyzed as follows: (g) anti-FasL, (h) anti-Fas, (i) anti-Bax, and (j) anti-Bcl2 $(n=5)$. The nuclei were stained with DAPI (blue). Scale bar $=50 \mu \mathrm{m}$. ${ }^{*} P<0.05 ;{ }^{* *} P<0.01 ; * * *<0.001$.
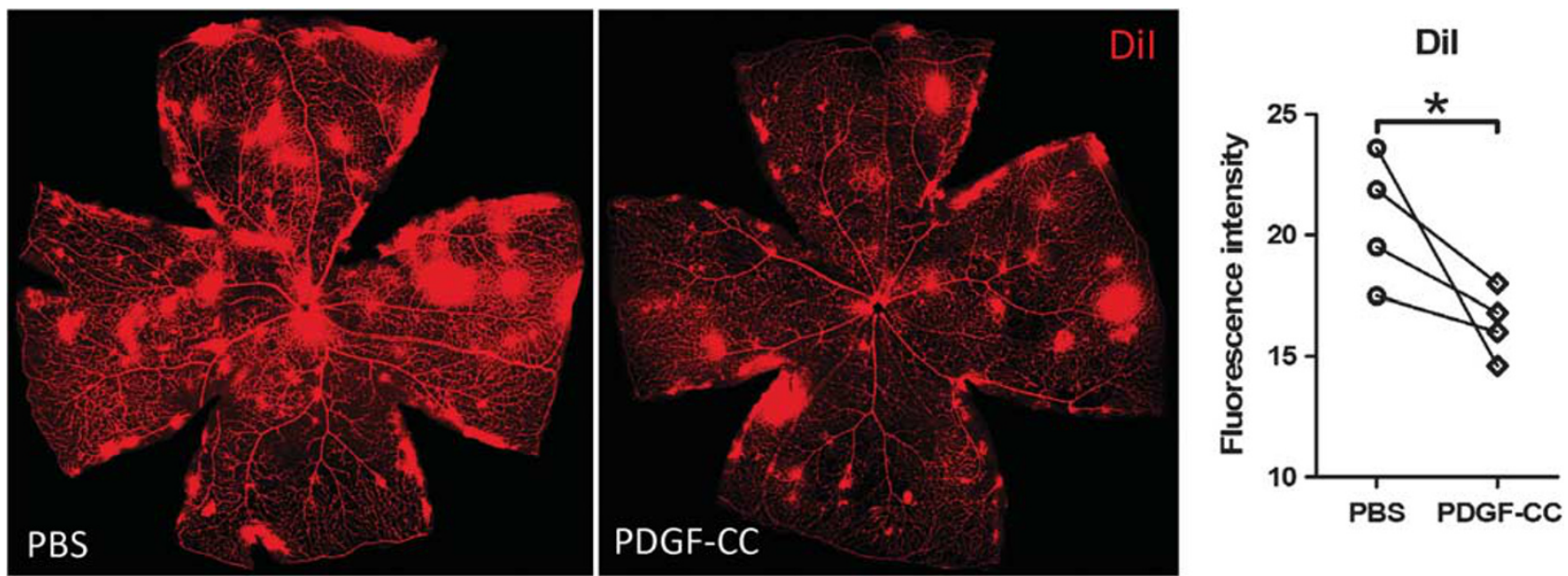

Figure 4 Effects of PDGF-C on retinal vessels in the neuroretina of DKO rd8 mice. Z-stacks of retinal vasculature stained with Dil showed less vessel leakage or vascularization in the PDGF-CC-treated than PBS-injected retina $(n=4)$. Image-J software is used to measure the fluorescence intensity in pixels per whole retina in each image. $* P<0.05$.

protein may serve to protect the neuroretina in DKO rd8 mice with AMD-like lesions and could be a potential therapeutic agent for treatment.
Maintenance of normal photoreceptor function is closely related with RPE based on its nutrient transportation for photoreceptors and phagocytosis of shed photoreceptor OSs. 
Lipofuscin accumulates as a byproduct of phagocytosis of photoreceptor OSs. Excessive levels of lipofuscin during aging can greatly compromise essential RPE functions and contribute to the AMD pathogenesis. ${ }^{46,49}$ Thus, A2E, the major component of lipofuscin, is regarded as a biomarker in AMD. ${ }^{46,50}$ Our study showed decreased A2E levels in 11 of 15 mice with PDGF-CC treatment compared with PBS controls. This suggests that PDGF-C has the effect to reduce an A2E level in the RPE of DKO $r d 8$ mice, which can preserve RPE, thus potentially help uphold the photoreceptor integrity.

One of the major causes of human AMD is photoreceptor apoptosis mediated by both extrinsic and intrinsic pathways. Upregulation of the FasL/Fas extrinsic pathway has been reported in human AMD specimens. ${ }^{34}$ Meanwhile, both the extrinsic FasL/Fas pathway and intrinsic Bcl2/Bax system are involved in photoreceptor/RPE apoptosis in DKO $r d 8$ mice. ${ }^{51,52}$ Our previous study demonstrated an elevation of FasL-associated photoreceptor apoptosis of DKO $r d 8$ mice than that of WT in physiological conditions. ${ }^{52}$ We also reported an upregulation of Bax transcript and a lower ratio of $B c l 2 / B a x$ in DKO $r d 8$ mouse eyes compared with WT. ${ }^{53}$ Interestingly, in DKO $r d 8$ eyes, PDGF-C decreased apoptotic retinal cells with lower FasL and Fas protein expressions. We did not detect a significant decrease of Fasl transcript in the DKO $r d 8$ retina with PDGF-CC treatment, which could be due to the discordant expression of transcript and protein of FasL in DKO $r d 8$ eyes or limited number of experimental animals. ${ }^{30,53}$ In the current study, PDGF-C significantly downregulated pro-apoptotic Bax protein and transcript levels. This finding is in agreement with the antiapoptotic effects of PDGF-C in the retina of optic nerve crush mouse model. ${ }^{12}$ In addition, we found an upregulation of antiapoptotic Bcl2 protein in PDGF-CC-treated DKO $r d 8$ eyes. Bcl2 confers negative control in the apoptosis pathway, and the actions of Bax in vivo are partially dependent on the formation of homo-oligomers. ${ }^{54,55}$ The cytoprotective mechanism of Bcl2 has been shown to involve in the formation of Bcl2/Bax heterodimer that prevents Bax oligomerization, which subsequently evokes a survival signal for the cells. ${ }^{54-56}$ Thus, the total effect of PDGF-C on Bcl2 family members is antiapoptotic. This effectively results in a survival trend in the DKO $r d 8$ retina.

We have previously reported that the PDGF-CC protein can activate both PDGFR- $\alpha$ and PDGFR- $\beta$ in the mouse retina. $^{12}$ Furthermore, their neutralizing antibodies can largely block the neuroprotective effect of PDGF-CC on retinal ganglion cells in vivo. The neuroprotective effect of PDGF-C was mediated by both PDGFR- $\alpha$ and PDGFR- $\beta$. We further identified that the neuroprotective effect of PDGF-C was achieved by regulating GSK $3 \beta$ phosphorylation and expression. GSK3 $\beta$ is a pro-apoptotic gene that has an important role in neuronal apoptosis. ${ }^{57}$ This is in accordance with our current study, showing the effect of PDGF-C on the neuroretina is via an antiapoptosis pathway.
Another important issue of PDGF-C treatment is the angiogenic effect on target tissues. Previous studies have shown that PDGF-C and PDGFRs are abundantly expressed by a variety of vascular cells, including pericytes. ${ }^{7,9-11}$ The highest density of pericytes in the body is found in vessels of the neural tissues, including the brain and retina. ${ }^{58}$ Pericytes have an important role in the integrity of structural vessels and the blood-retinal barriers. ${ }^{58,59}$ Thus, pericyte abnormality and degeneration would result in vascular defects. PDGF-C has a critical role in promoting blood vessel growth and maturation, and one of the target routes is through pericytes. ${ }^{16,17}$ It also induces new blood vessel formation by increasing the number and integrity of pericytes. ${ }^{60}$ PDGF-C drives the recruitment and differentiation of pericytes, resulting in better basement membrane integrity and a more mature, stable vascular wall with a lower permeability. ${ }^{61}$ Thus, with the supportive effect of PDGF-C on pericytes, the treated mice revealed better-preserved pericytes in the retinal vessels with PDGF-CC treatment than PBS controls. It also guarantees the relatively normal vessel function to provide blood supply for the retinal neurons, which contributes to PDGF-Cs' neuroprotective effects. More importantly, under the effective dosage for neuroprotection in this study, less vascular leakage and no neovascularization were noted in any of the PDGF-CCtreated eyes.

In summary, our data show that local application of PDGF-CC protein stabilizes focal photoreceptor degeneration in DKO $r d 8$ mice. The mechanism underlying the protective role is through the antiapoptotic pathway. PDGF-CC treatment under the effective neuroprotective dosage does not damage vascular pericytes, thus avoiding retinal neovascularization in this mouse model. The neuroprotective and survival effects of PDGF-C may provide a novel treatment for retinal degeneration diseases, such as AMD.

Supplementary Information accompanies the paper on the Laboratory Investigation website (http://www.laboratoryinvestigation.org)

\section{ACKNOWLEDGMENTS}

The National Eye Institute Intramural Research Program supported the study.

\section{DISCLOSURE/CONFLICT OF INTEREST}

The authors declare no conflict of interest.

1. Li X, Ponten $\mathrm{A}$, Aase $\mathrm{K}$, et al. PDGF-C is a new protease-activated ligand for the PDGF alpha-receptor. Nat Cell Biol 2000;2:302-309.

2. Kazlauskas A. A new member of an old family. Nat Cell Biol 2000;2:E78-E79.

3. Li X, Eriksson U. Novel PDGF family members: PDGF-C and PDGF-D. Cytokine Growth Factor Rev 2003;14:91-98.

4. Fredriksson L, Ehnman M, Fieber C, et al. Structural requirements for activation of latent platelet-derived growth factor CC by tissue plasminogen activator. J Biol Chem 2005;280:26856-26862.

5. Lee $C$, Zhang F, Tang Z, et al. PDGF-C: a new performer in the neurovascular interplay. Trends Mol Med 2013;19:474-486. 
6. Cao R, Brakenhielm E, Li X, et al. Angiogenesis stimulated by PDGF-CC a novel member in the PDGF family, involves activation of PDGFRalphaalpha and -alphabeta receptors. FASEB J 2002;16:1575-1583.

7. Gilbertson DG, Duff ME, West JW, et al. Platelet-derived growth factor C (PDGF-C), a novel growth factor that binds to PDGF alpha and beta receptor. J Biol Chem 2001;276:27406-27414.

8. Ding $\mathrm{H}, \mathrm{Wu} X$, Bostrom $\mathrm{H}$, et al. A specific requirement for PDGF-C in palate formation and PDGFR-alpha signaling. Nat Genet 2004;36: 1111-1116.

9. Karvinen $\mathrm{H}$, Rutanen $\mathrm{J}$, Leppanen $\mathrm{O}$, et al. PDGF-C and -D and their receptors PDGFR-alpha and PDGFR-beta in atherosclerotic human arteries. Eur J Clin Invest 2009:39:320-327.

10. Su EJ, Fredriksson L, Geyer M, et al. Activation of PDGF-CC by tissue plasminogen activator impairs blood-brain barrier integrity during ischemic stroke. Nat Med 2008;14:731-737.

11. Crawford Y, Kasman I, Yu L, et al. PDGF-C mediates the angiogenic and tumorigenic properties of fibroblasts associated with tumors refractory to anti-VEGF treatment. Cancer Cell 2009;15:21-34.

12. Tang $Z$, Arjunan $P$, Lee $C$, et al. Survival effect of PDGF-CC rescues neurons from apoptosis in both brain and retina by regulating GSK3beta phosphorylation. J Exp Med 2010;207:867-880.

13. Lei $H$, Hovland $P$, Velez $G$, et al. A potential role for PDGF-C in experimental and clinical proliferative vitreoretinopathy. Invest Ophthalmol Vis Sci 2007;48:2335-2342.

14. Hamada T, Ui-Tei K, Imaki J, et al. The expression of SCDGF/PDGF-C/ fallotein and SCDGF-B/PDGF-D in the rat central nervous system. Mech Dev 2002;112:161-164.

15. Li R, Maminishkis A, Wang FE, et al. PDGF-C and -D induced proliferation/migration of human RPE is abolished by inflammatory cytokines. Invest Ophthalmol Vis Sci 2007;48:5722-5732.

16. Li X, Tjwa M, Moons $\mathrm{L}$, et al. Revascularization of ischemic tissues by PDGF-CC via effects on endothelial cells and their progenitors. J Clin Invest 2005;115:118-127.

17. Greenberg Jl, Shields DJ, Barillas SG, et al. A role for VEGF as a negative regulator of pericyte function and vessel maturation. Nature 2008;456:809-813.

18. Hou X, Kumar A, Lee C, et al. PDGF-CC blockade inhibits pathological angiogenesis by acting on multiple cellular and molecular targets. Proc Natl Acad Sci USA 2010;107:12216-12221.

19. Coleman HR, Chan CC, Ferris 3rd FL, et al. Age-related macular degeneration. Lancet 2008;372:1835-1845.

20. Klein $\mathrm{R}$, Chou $\mathrm{CF}$, Klein $\mathrm{BE}$, et al. Prevalence of age-related macular degeneration in the US population. Arch Ophthalmol 2011;129:75-80.

21. Lim LS, Mitchell P, Seddon JM, et al. Age-related macular degeneration. Lancet 2012;379:1728-1738.

22. Bhutto I, Lutty G. Understanding age-related macular degeneration (AMD): relationships between the photoreceptor/retinal pigment epithelium/Bruch's membrane/choriocapillaris complex. Mol Aspects Med 2012;33:295-317.

23. Curcio CA, Medeiros NE, Millican CL. Photoreceptor loss in age-related macular degeneration. Invest Ophthalmol Vis Sci 1996;37:1236-1249.

24. Curcio CA. Photoreceptor topography in ageing and age-related maculopathy. Eye (Lond) 2001;15:376-383.

25. de Jong PT. Age-related macular degeneration. N Engl J Med 2006;355:1474-1485.

26. Colak E, Majkic-Singh N, Zoric $L$, et al. The impact of inflammation to the antioxidant defense parameters in AMD patients. Aging Clin Exp Res 2012;24:588-594.

27. $\mathrm{Xu} \mathrm{H}$, Chen $\mathrm{M}$, Forrester JV. Para-inflammation in the aging retina. Prog Retin Eye Res 2009;28:348-368.

28. Rutar M, Natoli R, Kozulin $\mathrm{P}$, et al. Analysis of complement expression in light-induced retinal degeneration: synthesis and deposition of C3 by microglia/macrophages is associated with focal photoreceptor degeneration. Invest Ophthalmol Vis Sci 2011;52:5347-5358.

29. Kauppinen A, Niskanen $H$, Suuronen $T$, et al. Oxidative stress activates NLRP3 inflammasomes in ARPE-19 cells-implications for age-related macular degeneration (AMD). Immunol Lett 2012;147:29-33.

30. Wang Y, Wang VM, Chan CC. The role of anti-inflammatory agents in age-related macular degeneration (AMD) treatment. Eye 2011;25 127-139.

31. Tarallo V, Hirano Y, Gelfand BD, et al. DICER1 loss and Alu RNA induce age-related macular degeneration via the NLRP3 inflammasome and MyD88. Cell 2012;149:847-859.
32. Ambati J, Fowler BJ. Mechanisms of age-related macular degeneration. Neuron 2012;75:26-39.

33. Ardeljan D, Chan CC. Aging is not a disease: Distinguishing age-related macular degeneration from aging. Prog Retin Eye Res 2013;37: 68-89.

34. Dunaief JL, Dentchev T, Ying GS, et al. The role of apoptosis in age-related macular degeneration. Arch Ophthalmol 2002;120: 1435-1442.

35. Yang P, Wiser JL, Peairs JJ, et al. Human RPE expression of cell survival factors. Invest Ophthalmol Vis Sci 2005;46:1755-1764.

36. Chu XK, Wang Y, Ardeljan D, et al. Controversial view of a genetically altered mouse model of focal retinal degeneration. Bioengineered 2012;4:130-135.

37. Ramkumar HL, Zhang J, Chan CC. Retinal ultrastructure of murine models of dry age-related macular degeneration (AMD). Prog Retin Eye Res 2010;29:169-190.

38. Tuo J, Bojanowski CM, Zhou M, et al. Murine $\mathrm{ccl} 2 / \mathrm{cx} 3 \mathrm{cr} 1$ deficiency results in retinal lesions mimicking human age-related macular degeneration. Invest Ophthalmol Vis Sci 2007;48:3827-3836.

39. Chan CC, Ross RJ, Shen D, et al. C $\mathrm{Cl} 2 / \mathrm{C} \times 3 \mathrm{Cr} 1$-deficient mice: an animal model for age-related macular degeneration. Ophthalmic Res 2008;40: 124-128.

40. Mattapallil MJ, Wawrousek EF, Chan CC, et al. The Rd8 mutation of the Crb1 gene is present in vendor lines of $\mathrm{C} 57 \mathrm{BL} / 6 \mathrm{~N}$ mice and embryonic stem cells, and confounds ocular induced mutant phenotypes. Invest Ophthalmol Vis Sci 2012;53:2921-2927.

41. Chen M, Hombrebueno JR, Luo $C$, et al. Age- and light-dependent development of localised retinal atrophy in CCL2(-/-)CX3CR1(GFP/GFP) mice. PLoS One 2013:8:e61381.

42. Yu CR, Lin JX, Fink DW, et al. Differential utilization of Janus kinasesignal transducer activator of transcription signaling pathways in the stimulation of human natural killer cells by IL-2, IL-12, and IFN-alpha. J Immunol 1996;157:126-137.

43. Tuo J, Pang JJ, Cao X, et al. AAV5-mediated sFLT01 gene therapy arrests retinal lesions in $\mathrm{Ccl} 2(-/-) / \mathrm{C} \times 3 \mathrm{Cr} 1(-/-)$ mice. Neurobiol Aging 2012;33:433e431-410

44. Tuo J, Cao X, Shen D, et al. Anti-inflammatory recombinant TSG-6 stabilizes the progression of focal retinal degeneration in a murine model. J Neuroinflammation 2012;9:59.

45. Li Y, Song $Y$, Zhao $L$, et al. Direct labeling and visualization of blood vessels with lipophilic carbocyanine dye Dil. Nat Protoc 2008;3 1703-1708.

46. Ben-Shabat S, Parish CA, Hashimoto M, et al. Fluorescent pigments of the retinal pigment epithelium and age-related macular degeneration. Bio-org Med Chem Lett 2001;11:1533-1540.

47. Karan G, Lillo C, Yang Z, et al. Lipofuscin accumulation, abnormal electrophysiology, and photoreceptor degeneration in mutant ELOVL4 transgenic mice: a model for macular degeneration. Proc Natl Acad Sci USA 2005;102:4164-4169.

48. Parish CA, Hashimoto $M$, Nakanishi $K$, et al. Isolation and one-step preparation of $A 2 E$ and iso-A2E, fluorophores from human retinal pigment epithelium. Proc Natl Acad Sci USA 1998;95:14609-14613.

49. Delori FC, Goger DG, Dorey CK. Age-related accumulation and spatial distribution of lipofuscin in RPE of normal subjects. Invest Ophthalmol Vis Sci 2001:42:1855-1866.

50. Wolf G. Lipofuscin and macular degeneration. Nutr Rev 2003; 61:342-346.

51. Ding $X$, Patel $M$, Shen $D$, et al. Enhanced HtrA2/Omi expression in oxidative injury to retinal pigment epithelial cells and murine models of neurodegeneration. Invest Ophthalmol Vis Sci 2009;50: 4957-4966.

52. Wang $Y$, Shen $D$, Wang VM, et al. Enhanced apoptosis in retinal pigment epithelium under inflammatory stimuli and oxidative stress. Apoptosis 2012;17:1144-1155.

53. Cao X, Liu M, Tuo J, et al. The effects of quercetin in cultured human RPE cells under oxidative stress and in $\mathrm{C} / 2 / \mathrm{C} \times 3 \mathrm{cr} 1$ double deficient mice. Exp Eye Res 2010;91:15-25.

54. Basu A, Haldar S. The relationship between $\mathrm{BCl} 2$, Bax and $\mathrm{p} 53$ : consequences for cell cycle progression and cell death. Mol Hum Reprod 1998:4:1099-1109.

55. Mikhailov V, Mikhailova M, Pulkrabek DJ, et al. Bcl-2 prevents Bax oligomerization in the mitochondrial outer membrane. J Biol Chem 2001;276:18361-18374. 
56. Ku B, Liang C, Jung JU, et al. Evidence that inhibition of BAX activation by $B C L-2$ involves its tight and preferential interaction with the BH3 domain of BAX. Cell Res 2011;21:627-641.

57. Liang $\mathrm{MH}$, Chuang DM. Regulation and function of glycogen synthase kinase-3 isoforms in neuronal survival. J Biol Chem 2007;282: 3904-3917.

58. Bergers $\mathrm{G}$, Song $\mathrm{S}$. The role of pericytes in blood-vessel formation and maintenance. Neuro Oncol 2005;7:452-464.
59. Bandopadhyay R, Orte C, Lawrenson JG, et al. Contractile proteins in pericytes at the blood-brain and blood-retinal barriers. J Neurocytol 2001;30:35-44.

60. Li X, Kumar A, Zhang F, et al. VEGF-independent angiogenic pathways induced by PDGF-C. Oncotarget 2010;1:309-314.

61. di Tomaso E, London N, Fuja D, et al. PDGF-C induces maturation of blood vessels in a model of glioblastoma and attenuates the response to anti-VEGF treatment. PLoS ONE 2009;4:e5123. 